\title{
A THREE-DIMENSIONAL BACKWARD LAGRANGIAN FOOTPRINT MODEL FOR A WIDE RANGE OF BOUNDARY-LAYER STRATIFICATIONS
}

\author{
N. KLJUN ${ }^{1, \star}$, M. W. ROTACH${ }^{1}$ and H. P. SCHMID ${ }^{2}$ \\ ${ }^{1}$ Institute for Atmospheric and Climate Science ETH, Zurich, Switzerland; ${ }^{2}$ Department of \\ Geography, Indiana University, Bloomington, Indiana, U.S.A.
}

(Received in final form 10 September 2001)

\begin{abstract}
We present a three-dimensional Lagrangian footprint model with the ability to predict the area of influence (footprint) of a measurement within a wide range of boundary-layer stratifications and receptor heights. The model approach uses stochastic backward trajectories of particles and satisfies the well-mixed condition in inhomogeneous turbulence for continuous transitions from stable to convective stratification. We introduce a spin-up procedure of the model and a statistical treatment of particle touchdowns which leads to a significant reduction of CPU time compared to conventional footprint modelling approaches. A comparison with other footprint models (of the analytical and Lagrangian type) suggests that the present backward Lagrangian model provides valid footprint predictions under any stratification and, moreover, for applications that reach across different similarity scaling domains (e.g., surface layer to mixed layer, for use in connection with aircraft measurements or with observations on high towers).
\end{abstract}

Keywords: Backward trajectories, Boundary-layer stability, Density kernel estimation, Lagrangian particle model, Source area, Spin-up.

\section{Introduction}

Common micrometeorological techniques for determining the trace gas concentration or flux at a fixed receptor downwind of a source give little information about the source location and size. Moreover, these concentrations and fluxes vary as a result of the turbulence state of the planetary boundary layer. Thus, a quantitative description of the surface flux budgets of atmospheric trace gases on a regional scale is far from trivial, as any source near the ground could potentially contribute to the measured concentration or flux.

In order to describe the area of influence to a measurement, Schmid and Oke (1990) define a 'source area' as that part of the upwind area which contains the effective sources and sinks contributing to a given measurement point. The term 'footprint' is specified as the relative contribution from each element of the upwind surface area source to the measured concentration or vertical flux (Schuepp et al., 1990). It can be interpreted as the probability that trace gas emitted from a

^ E-mail: nkljun@ethz.ch 
given elemental source reaches the measurement point. Functions describing the relationship between the spatial distribution of surface sources (or sinks) and a measured signal have been termed the 'footprint function' or the 'source weight function' (Schmid, 1994).

In recent years, several models have been proposed to estimate the size of the footprint and its dependence on the height of the measurement, wind velocity, surface roughness, heterogeneous underlying surfaces, and atmospheric stability. Basically, a footprint can be derived using three different methods: Lagrangian stochastic dispersion models, in most cases assuming Gaussian turbulence (e.g., Leclerc and Thurtell, 1990; Horst and Weil, 1992; Flesch et al., 1995; Rannik et al., 2000), analytical solutions of the diffusion equation by applying a K-theory model (e.g., Schuepp et al., 1990; Schmid and Oke, 1990; Wilson and Swaters, 1991; Horst and Weil, 1992) or large eddy simulation (e.g., Leclerc et al., 1997). See Schmid (2001) for a review.

Ideally, a footprint model is expected to yield information for the design of experiments under various environmental and experimental conditions (i.e., different measurement heights, atmospheric stability, and surface properties), and for the correct interpretation of measurement results. However, none of the analytical footprint models is valid outside the surface layer, whereas most of the Lagrangian particle models used for footprint modelling fulfill the well-mixed condition (Thomson, 1987) for only one given stability regime. On the other hand, large eddy simulation (LES) is highly CPU time intensive, and is notoriously weak in predicting the eddy structure close to the surface.

The present footprint model is based on the Lagrangian stochastic particle dispersion model developed by Rotach et al. (1996) in its two-dimensional version, and later extended to three dimensions by de Haan and Rotach (1998). This model satisfies the well-mixed condition continuously for stable to convective conditions, as well as for receptors above the surface layer (e.g., for use in connection with aircraft measurements as done in Schuepp et al., 1990, and others). The model employs a recently established approach using stochastic backward trajectories of particles (Flesch et al., 1995). This approach allows one to calculate the footprint for a measurement point instead of an average over a sensor volume without a coordinate transformation that requires horizontal homogeneity of the flow. In spatially inhomogeneous flow, 'forward' (dispersion) models need to simulate a potentially large number of sources, to resolve the inhomogeneity. Their computational expense is proportional to the number of sources needed (Luhar and Rao, 1994).

The following section describes a backward-trajectory model, and its application in the determination of a flux or concentration footprint. The sensitivity of the footprint to the initial velocities and the atmospheric stability is discussed in Section 3. Finally, in Section 4, the approach is evaluated indirectly by comparing the model results with corresponding estimates of the analytical footprint models 
FSAM and SAM of Schmid (1997), based on Horst and Weil (1992), as well as with another footprint model of the Lagrangian type after Rannik et al. (2000).

\section{Model Description}

\subsection{The LAGRANGiAn STOCHASTIC PARTIClE MODEL}

The Lagrangian formulation assumes that the diffusion of a passive scalar released from a surface source is statistically equivalent to the dispersion of an ensemble of particles that impact the ground within the surface source and thereafter 'carry' the scalar. Each of those 'marked' particles moves independently of all the others and is advected downwind by the mean flow and dispersed by turbulence. The diffusion of the scalar is described by a stochastic differential equation (the generalised Langevin equation), which determines the evolution of a Lagrangian particle at position $\mathbf{x}=(x, y, z)$ with the particle velocity $\mathbf{u}=\left(\bar{u}+u^{\prime}, v^{\prime}, w^{\prime}\right)$ in space and time (Thomson, 1987)

$$
\begin{aligned}
\mathrm{d} u_{i}^{\prime} & =a_{i}(\mathbf{x}, \mathbf{u}, t) \mathrm{d} t+b_{i j}(\mathbf{x}, \mathbf{u}, t) \mathrm{d} \xi_{j} \\
\mathrm{~d} \mathbf{x} & =\mathbf{u} \mathrm{d} t
\end{aligned}
$$

The functions $a_{i}$ determine the correlated part depending on turbulent velocity components, functions $b_{i j}$ the uncorrelated random contribution of the acceleration of the velocity components $u_{i}^{\prime}(i=1,2,3)$ with the increments $\mathrm{d} \xi_{j}$ from a Gaussian distribution with zero mean and variance $\mathrm{d} t$.

The well-mixed condition (Thomson, 1987) implies that the Fokker-Planck equation is an Eulerian form of (1) and can be used to determine the functions $a_{i}$ and $b_{i j}$ of Equation (1). For stationary turbulence, the corresponding Fokker-Planck equation can be written

$$
a_{i} P_{\mathrm{tot}}=\frac{\partial}{\partial u_{j}^{\prime}}\left(B_{i j} P_{\mathrm{tot}}\right)+\Phi_{i}
$$

where $P_{\text {tot }}$ denotes the probability density function (PDF) of the particle velocities, and (applying Einstein's summation convention)

$$
\begin{aligned}
& 2 B_{i j}=b_{i k} b_{j k} \\
& \frac{\partial \Phi_{i}}{\partial u_{i}^{\prime}}=-\frac{\partial}{\partial x_{i}}\left(u_{i}^{\prime} P_{\mathrm{tot}}\right) .
\end{aligned}
$$

We follow Thomson (1987) in using the behaviour of the structure function in the inertial subrange to specify $B_{i j}$. Thomson (1987) shows that $B_{i j}$ is non-zero only for $i=j$. Thus, we use $B_{i i}=B_{*}=\frac{1}{2} C_{0} \varepsilon$ (here, the summation convention 
does not apply). $C_{0}$ is a universal constant for the structure function, taken to be 3 in the present simulations (Rotach et al., 1996; Du, 1995, 1997) and $\varepsilon$ is the dissipation rate of turbulence kinetic energy. Since $B_{*}$ is not an explicit function of $\mathbf{u}$, Equation (2) can be rewritten as

$$
a_{i}=\frac{1}{P_{\mathrm{tot}}}\left(-B_{*} Q_{i}+\Phi_{i}\right)
$$

with

$$
Q_{i}=-\frac{\partial P_{\mathrm{tot}}}{\partial u_{i}^{\prime}} .
$$

The conventional approach of using a Lagrangian model for footprint estimates is to release particles at the surface source and track their trajectories until they have passed the receptor location. The footprint can then be computed after a coordinate transformation that requires horizontal homogeneity of the flow. Alternatively, it is possible to calculate the trajectories of a Lagrangian model in a backward time frame. In this case, the trajectories are initiated at the measurement point itself and are tracked backward in time, with a negative time step, from the sensor to any potential surface source (cf. Flesch et al., 1995). The advantage of this approach (in the following denoted as LPDM-B) is that only the trajectories that pass exactly through the measurement point need to be followed and therefore all of the calculated trajectories can be used directly and without coordinate transformation. In principle, the backward approach can consider sources at arbitrary levels or geometries with one single simulation, but is specific to a given measurement height, in contrast to the forward approach.

Thomson (1987) stated that the only change required to adapt the Langevin equation for reverse diffusion is a sign change of the first term of the coefficient $a_{i}$ in Equation (5). Therefore, for the LPDM-B, the derivative $Q_{i}$ in Equation (6) is modified to

$$
Q_{i}=+\frac{\partial P_{\mathrm{tot}}}{\partial u_{i}^{\prime}} .
$$

Turbulence is not generally a Gaussian process, but for many applications, the assumption of Gaussian turbulent diffusion is an acceptable approximation. A major exception is the convective boundary layer (CBL) where vertical dispersion is dramatically different from that in neutral or stable boundary layers since large coherent updrafts and downdrafts dominate the vertical flow statistics. Therefore, the PDF of the vertical velocity in the CBL is non-Gaussian. Lagrangian stochastic modelling is able to account for the effects of skewed velocity distributions. In the CBL, updrafts have higher vertical velocities but occupy less area than downdrafts, and thus the skewness is positive in a forward time frame. Due to the reverse 
time development in the LPDM-B approach, the corresponding vertical velocity skewness is also reversed.

Baerentsen and Berkowicz (1984) proposed to approximate the skewed vertical velocity PDF of the CBL by the sum of two Gaussian distributions, one for the updrafts and the other for the downdrafts. This technique was adopted by Rotach et al. (1996). The skewed PDF of the vertical velocity $P_{S}$ for the 'backward' model may then be written as

$$
P_{S}=C_{\text {up }} P_{\text {up }}+C_{\text {down }} P_{\text {down }} .
$$

The parameters $C_{\text {up }}$ and $C_{\text {down }}$ determine the relative strengths of updrafts and downdrafts with $C_{\text {up }}=1-C_{\text {down }}$. The two Gaussian distributions are

$$
\begin{aligned}
P_{\text {up }} & =\frac{1}{\sqrt{2 \pi} \sigma_{\text {up }}} \exp \left\{-\frac{1}{2}\left(\frac{w+\bar{w}_{\text {up }}}{\sigma_{\text {up }}}\right)^{2}\right\} \\
P_{\text {down }} & =\frac{1}{\sqrt{2 \pi} \sigma_{\text {down }}} \exp \left\{-\frac{1}{2}\left(\frac{w-\bar{w}_{\text {down }}}{\sigma_{\text {down }}}\right)^{2}\right\} .
\end{aligned}
$$

To account for the whole range of boundary-layer stabilities, $P_{\text {tot }}$ is modelled using a transition function $\mathcal{F}$, a continuous function of the boundary-layer height $z_{i}$, the stability within the boundary layer, $L$, and the height $z$ of each particle, following Rotach et al. (1996). $\mathcal{F}$ is specified to approach 1 under fully convective conditions (sufficiently far above the surface) and $\mathscr{F} \rightarrow 0$ under near-neutral or stable stratification and when approaching the surface. For details see Rotach et al. (1996). In this fashion, a continuous PDF from skewed to Gaussian, $P_{\text {tot }}$, is given by

$$
P_{\mathrm{tot}}=(1-\mathcal{F}) P_{G}+\mathcal{F} P_{u} P_{v} P_{S},
$$

where $P_{S}$ is given by (8) and

$$
\begin{aligned}
P_{G} & =P_{u} P_{v} P_{w} P_{u w} \\
P_{u_{i}} & =\frac{1}{\sqrt{2 \pi} \sigma_{u_{i}}} \exp \left\{-\frac{u_{i}^{\prime 2}}{2\left(1-\rho^{2}\right) \sigma_{u_{i}}^{2}}\right\} \\
P_{u w} & =\frac{1}{\sqrt{1-\rho^{2}}} \exp \left\{-\frac{\rho}{\left(1-\rho^{2}\right) \sigma_{u} \sigma_{w}} u^{\prime} w^{\prime}\right\},
\end{aligned}
$$

and $\rho=\overline{u^{\prime} w^{\prime}} /\left(\sigma_{u} \sigma_{w}\right)$ is the correlation coefficient of streamwise and vertical velocities. Thus, in the convective limit, the PDF (Equation (10)) is skewed in the vertical velocity and the components are independent of each other. On the other hand, in neutral or stable conditions, $u^{\prime}$ and $w^{\prime}$ are correlated and Gaussian. 
For an appropriate evolution of the particle trajectories, several turbulence parameterisations need to be considered, namely the profiles of mean wind speed, Reynolds stress, the dissipation rate of turbulence kinetic energy, the particle velocity variances, the vertical velocity skewness, and the constants $C_{\text {up }}$ and $C_{\text {down }}$. These parameterisations have been validated within the forward Lagrangian dispersion model (LPDM) for fully convective, forced convective and ideally neutral conditions. For a complete description of the model development, its turbulence parameterisations, and the validation of the dispersion model, the reader is referred to Rotach et al. (1996).

\subsection{THE FOOTPRINT FOR CONCENTRATION AND FLUX ESTIMATES}

For the determination of footprint distributions in the backward mode, an ensemble of $n$ particle trajectories is initiated at the receptor location $P\left(x, y, z_{m}\right)$ with random initial velocities $\mathbf{u}_{i n i}$. Most of the particles touch the ground (represented by reflection at a small height, $z_{r}$ ), once or several times with a vertical velocity $w_{t}$ (touchdown velocity) at various points $\left(x_{t}, y_{t}\right)$, most of them upwind. Throughout the simulation, all touchdown locations and velocities are stored to be used in the footprint estimation following Flesch (1996).

A surface area source with uniform emission rate of strength $Q\left(\mathrm{~kg} \mathrm{~m}^{-2} \mathrm{~s}^{-1}\right)$ can be viewed as a thin volume extending an infinitesimal height $\mathrm{d} z$ above the ground $\left(V_{s r c}=A_{s r c} \mathrm{~d} z\right.$ ), with an equivalent volumetric emission rate $S=Q / \mathrm{d} z$ (Flesch et al., 1995). Each particle $i$ that touches the ground within the source with a vertical velocity $w_{t}^{i}$ has a residence time 'within' the source of

$$
T_{i}=2 \frac{\mathrm{d} z}{w_{t}^{i}}
$$

as it passes the source volume on its impact as well as after the reflection on the ground. The contribution of this particle to the concentration increment at the receptor is then

$$
\Delta C_{i}(\mathbf{x})=S \cdot T_{i}=2 \frac{Q}{w_{t}^{i}} .
$$

For $n$ released particles, the concentration at the receptor can be written as (cf. Flesch et al., 1995)

$$
C(\mathbf{x})=S \frac{1}{n} \sum_{i=1}^{n_{t}} 2 \frac{\mathrm{d} z}{w_{t}^{i}}=\frac{Q}{n} \sum_{i=1}^{n_{t}} \frac{2}{w_{t}^{i}},
$$

where the summation refers to all touchdowns $\left(n_{t}\right)$ assuming an extended surface source. For the case where the spatial extent of a surface source is known, the summation may run over touchdowns within this source only. 
The determination of the vertical flux density for the source requires only a simple variation of the method described above. The flux increment at the receptor caused by a single particle $i$ touching down within a source may be given by

$$
\Delta F_{i}(\mathbf{x})=\Delta C_{i}(\mathbf{x}) \cdot w_{i n i}^{i},
$$

using the vertical velocity of the particle at the receptor location (in a backward model, this is of course the initial velocity of the particle, $w_{i n i}^{i}$ ).

Substituting the concentration increment of Equation (15) into Equation (17) and considering all $n$ released particles yields the vertical flux density estimation at the receptor location $P\left(x, y, z_{m}\right)$ for a sustained surface source $Q$ (cf. Flesch, 1996)

$$
F=2 \frac{Q}{n} \sum_{i=1}^{n_{t}} \frac{w_{i n i}^{i}}{w_{t}^{i}}
$$

Again, the summation runs over all particle touchdowns, $w_{i n i}$ and $w_{t}$ are the vertical initial and touchdown velocities, respectively, corresponding to each individual particle touchdown. Special attention needs to be paid to the distribution of the initial vertical velocities, as it directly influences the flux estimate. The sensitivity of the footprint to this velocity distribution is examined in Section 3.1.

Additionally, with given downstream concentration or flux measurements, the emission rate of a surface-area source can be estimated using Equation (16) or (18) (Flesch et al., 1995).

The concentration footprint $f_{c}$ and the flux footprint $f_{f}$ can be determined using the touchdown positions and velocities provided by the LPDM-B by simply calculating

$$
\begin{aligned}
f_{c} & =\frac{1}{Q} \frac{\partial^{2} C}{\partial x \partial y} \\
f_{f} & =\frac{1}{Q} \frac{\partial^{2} F}{\partial x \partial y},
\end{aligned}
$$

and for the crosswind-integrated footprint

$$
\begin{aligned}
& {\overline{f_{c}}}^{y}=\frac{1}{Q} \frac{\partial C}{\partial x} \\
& {\overline{f_{f}}}^{y}=\frac{1}{Q} \frac{\partial F}{\partial x} .
\end{aligned}
$$




\subsection{DENSITY KERNEL ESTIMATION}

The traditional way of evaluating the footprint is to overlay the upwind area with a grid and sum the footprint value of the particle touchdowns within each grid cell. Using this method, the footprint strongly depends on the selected grid spacing and on the number of particles released in the simulation. Here, we propose a statistical treatment of the particle touchdowns (Kljun et al., 2000a), using the density kernel method after de Haan (1999) who investigates this approach in detail for dispersion modelling.

The footprint values of the touchdowns are treated as data points and a distribution (kernel function $K$ ) is attached to each data point, to express the touchdown density in a point, rather than over a discrete grid-cell. These distributions may be Gaussian or Epanechnikov, bi-, tri-, quad- and quint-weight kernels. In the present study, we use biweight kernels according to

$$
K(\mathbf{r})=\left\{\begin{array}{cl}
C_{2}\left(1-\mathbf{r} \cdot \mathbf{r}^{T}\right)^{2} & \left(\mathbf{r} \cdot \mathbf{r}^{T}<1\right) \\
0 & \text { otherwise }
\end{array}\right.
$$

with

$$
\int K(\mathbf{r}) \mathrm{d} \mathbf{r}=1,
$$

where $\mathbf{r}$ is a d-dimensional vector and $C_{2}$ a normalising factor (cf. de Haan, 1999). We then evaluate and sum the values of those distributions weighted by $f_{i}$ at any selected point $\mathbf{x}$ in space to get

$$
f(\mathbf{x})=\sum_{i=1}^{n_{t}} \frac{f_{i}}{h_{i}^{2}} K\left(\frac{\mathbf{x}-\mathbf{x}_{i}}{h_{i}}\right),
$$

where $n_{t}$ is the total number of touchdowns and $h$ is the size (bandwidth) of the kernels. For the optimal specification of $h$, see de Haan (1999).

A so-called locally optimised density kernel estimation is performed. A firstguess density estimate is computed using the same global kernel size, $h$, for all touchdown locations. Then, an optimised density estimation using individual kernel sizes, $h_{i}$, for each data point (i.e., touchdown location) is performed. We decrease the bandwidth of the kernels if the data points are close to each other (i.e., the first-guess kernel is high), and increase the bandwidth in areas with a low first-guess density. This way, a continuous distribution adjusted to the touchdown density is derived. A grid-box average, on the other hand, would lead to a resolution of the footprint that is not adjusted to the touchdown density. The discrete distribution of the grid-box average requires smoothing, further decreasing the resolution.

The main advantages of the density kernel estimation method are the lack of the need to choose (more or less arbitrary) values for the size and location of the 
averaging grid boxes, the higher efficiency due to the locally optimised kernel sizes, and the higher resolution as compared to grid-box averaging for identical numbers of data points. Therefore, it is possible to decrease the number of simulated particles to something between $1-5 \times 10^{3}$ particles (compared to $5 \times 10^{4}$ particles necessary when using a grid-box average to obtain a similar footprint resolution). This decrease of course results in a significant reduction of CPU-time by more than an order of magnitude. Typically, a simulation of LPDM-B applying the density kernel method needs 5 to 15 minutes on a Sun Ultra60 workstation.

\section{Sensitivity Analysis}

\subsection{INITIAL VELOCITIES}

The simulated flux footprint depends strongly on the initial velocities since the initial velocities are explicitly included in the footprint calculation (Equation (18)). However, using Lagrangian particle models, it is not straightforward to create appropriate initial velocity distributions for any atmospheric stability. Often, the correlation of the streamwise and vertical velocity components is neglected when creating them. As a result, unrealistic individual particle velocities may be produced, with detrimental consequences for the evaluation of Equation (18).

A very simple solution to this problem is the introduction of a spin-up routine (after T. Flesch, personal communication, 2001): At first, velocities are assigned to each of the particles from a sample that approximates the atmospheric velocity PDF at the sensor location. During the spin-up, the evolution of the particle velocities is calculated as usual, according to the first part of Equation (1), but the incremental displacement in the second part is ignored $(\mathrm{d} \mathbf{x}=0)$, so that the particles are kept at their original position. Note that this procedure implies that all the involved spatial derivatives must be forced to zero because the particles are not 'allowed' to move. Thus, when the particles finally leave the receptor after the spinup, unrealistic velocity distributions of the initial approximate PDF are smoothed out and almost perfect density distributions of initial velocities are reached. While the distributions without spin-up were almost symmetric (neglected correlation of $u^{\prime}$ and $w^{\prime}$ ), the spin-up procedure leads to a more realistic distribution between the flow quadrants (Figure 1). It was found that a spin-up of about 1000 updates of the particle velocities is sufficient over the range of boundary-layer stabilities and receptor heights simulated here.

Moreover, the modelled velocity distributions were compared to distributions as obtained from observations. For this purpose, ten half-hourly periods with stable $(z / L \approx 1)$ and near-neutral $(z / L \approx 0.03)$ conditions were selected from the data of Forrer and Rotach (1997), and ten periods of convective stability $(z / L \approx-1)$ were considered from observations over a flat agricultural site in Switzerland. Table I shows a quadrant analysis of the fluctuating components as introduced by Shaw 


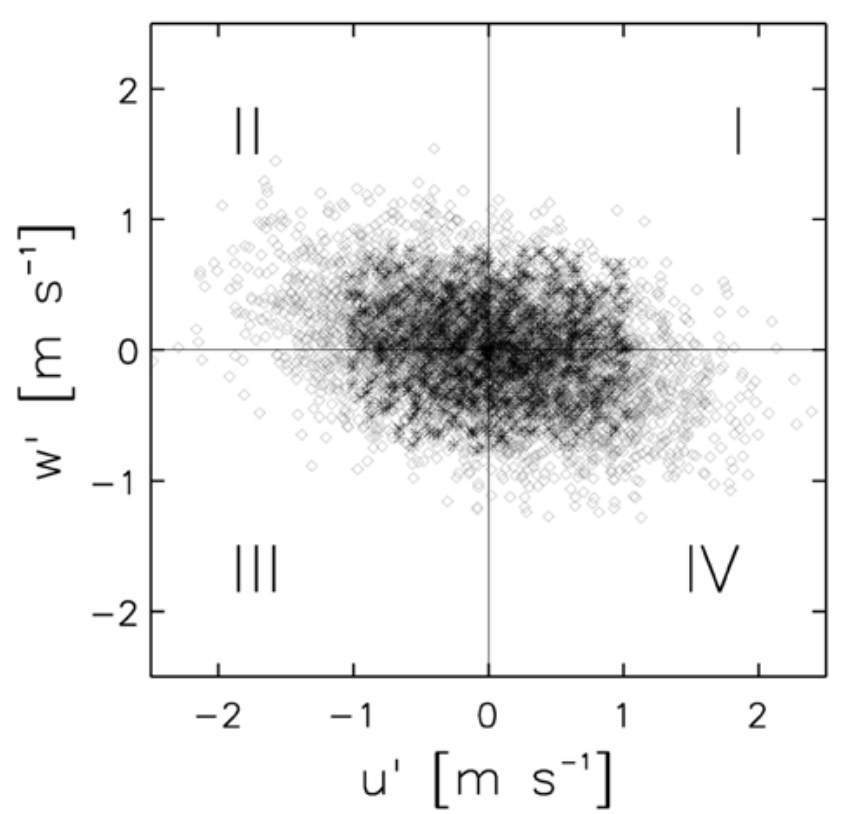

Figure 1. Distribution of the fluctuating components $w^{\prime}$ against $u^{\prime}$ with $(\diamond)$ and without $(*)$ spin-up under near-neutral stratification with $z / L=0.03$. I: outward interaction, II: ejection, III: inward interaction, IV: sweep, referring to the 'forward' terminology.

et al. (1983). The stress fractions contributed by each quadrant (referring to the 'forward' terminology; I: outward interaction, II: ejection, III: inward interaction, IV: sweep, cf. Figure 1) predicted by the model with spin-up were compared to the corresponding measured distributions. Very good correspondence to the measured velocity distributions was obtained by the spin-up method. Without spin-up, the correlation between horizontal and vertical velocity components vanishes (Figure 1) and thus a quadrant analysis becomes problematic. However, it is clear from Figure 1 that neither the quadrant contribution nor the total flux can be correct for the velocity distributions without spin-up.

How does the spin-up affect the footprint estimate? Figure 2 compares a footprint simulation using the velocity distribution after a spin-up and a simulation with the raw initial velocities without spin-up. The position of the maximum influence (peak of the footprint), as well as its magnitude, was significantly altered by starting with an imperfect velocity distribution. Comparing footprint simulations with and without spin-up under different stability-regimes (convective, neutral, stable), the peak distance of the footprint was typically $30 \%$ smaller with spin-up. This difference clearly demonstrates the importance of the correct correlation of the initial velocity components.

All simulations presented in the following were run with spin-up. 


\section{TABLE I}

Quadrant analysis for the fluctuating components $w^{\prime}$ and $u^{\prime}\left(\overline{u^{\prime} w^{\prime}}(\right.$ Quadrant $\left.) / \overline{u^{\prime} w^{\prime}}\right)$ derived from observations and from the LPDM-B with spin-up (cf. Figure 1). Units of $\mathrm{m}^{2} \mathrm{~s}^{-2}$.

\begin{tabular}{|c|c|c|c|c|c|c|}
\hline & \multicolumn{2}{|c|}{ Stable: $z / L \approx 1$} & \multicolumn{2}{|c|}{ Near-neutral: $z / L \approx 0.03$} & \multicolumn{2}{|c|}{ Convective: $z / L \approx-1$} \\
\hline & Observation & LPDM-B & Observation & LPDM-B & Observation & LPDM-B \\
\hline I & $-0.48 \pm 0.10$ & -0.39 & $-0.60 \pm 0.13$ & -0.56 & $-0.66 \pm 0.03$ & -0.67 \\
\hline II & $1.07 \pm 0.13$ & 0.86 & $1.12 \pm 0.07$ & 1.06 & $1.25 \pm 0.13$ & 1.16 \\
\hline III & $-0.49 \pm 0.08$ & -0.38 & $-0.53 \pm 0.09$ & -0.54 & $-0.68 \pm 0.14$ & -0.65 \\
\hline IV & $0.90 \pm 0.09$ & 0.91 & $1.02 \pm 0.22$ & 1.04 & $1.09 \pm 0.05$ & 1.16 \\
\hline$\overline{u^{\prime} w^{\prime}}$ & $-0.13 \pm 0.01$ & -0.12 & $-0.26 \pm 0.04$ & -0.23 & $-0.11 \pm 0.08$ & -0.04 \\
\hline
\end{tabular}

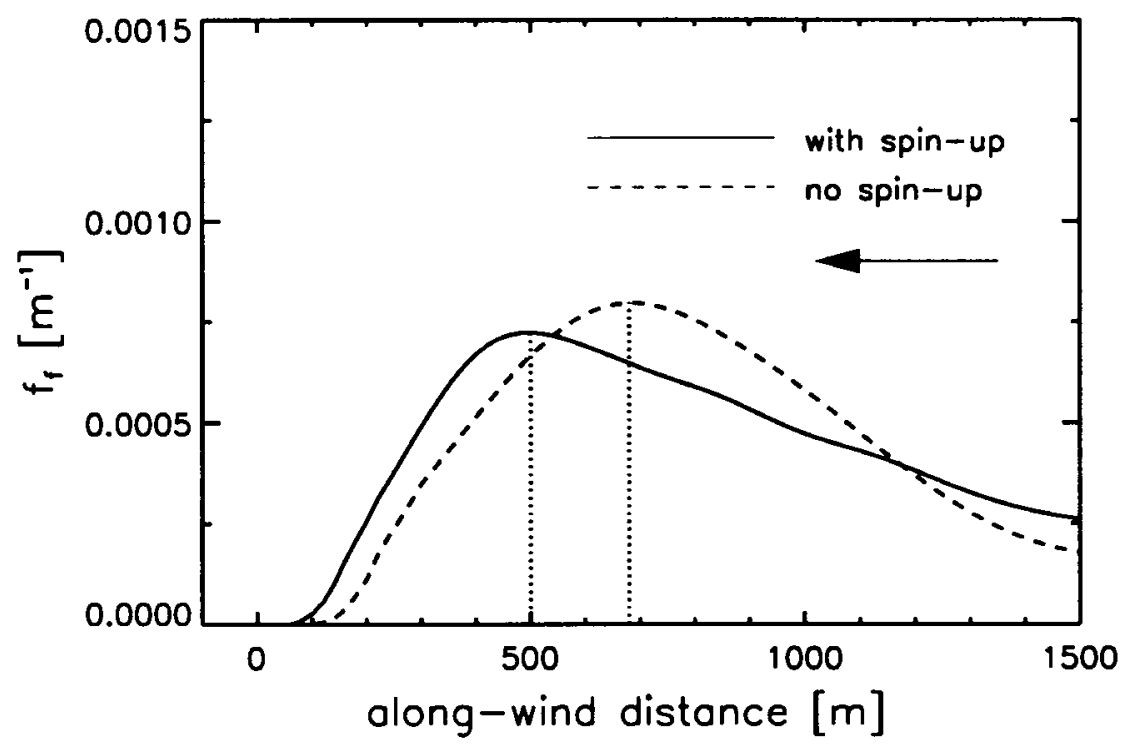

Figure 2. Crosswind-integrated flux footprint as predicted with and without using a spin-up under stable stratification (cf. Table II). The arrow indicates the wind direction.

\subsection{BOUNDARY-LAYER STABILITY}

As is well-known, the footprint is sensitive to the stability. With its ability to predict footprint estimates for a broad range of boundary-layer stabilities, the present model is well-suited to reveal this sensitivity. For this reason, four typical cases of stability were selected as described in Table II and the corresponding flux and concentration footprints were calculated for a receptor height of $50 \mathrm{~m}$.

Figure 3 shows the crosswind-integrated footprints predicted by the Lagrangian model. It can be seen that, for both footprint types, the peak location varies with 
TABLE II

Velocity scales, Obukhov length, and boundary-layer height describing the stabilities for four different simulations.

\begin{tabular}{lllll}
\hline & $\mathrm{u}_{*}\left(\mathrm{~m} \mathrm{~s}^{-1}\right)$ & $\mathrm{w}_{*}\left(\mathrm{~m} \mathrm{~s}^{-1}\right)$ & $\mathrm{L}(\mathrm{m})$ & $\mathrm{z}_{i}(\mathrm{~m})$ \\
\hline Strongly convective & 0.2 & 2.0 & -5 & 2000 \\
Forced convective & 0.2 & 1.0 & -36 & 1800 \\
Neutral & 0.8 & - & $\infty$ & 1500 \\
Stable & 0.5 & - & 100 & 190 \\
\hline
\end{tabular}

Receptor at $(0,0,50) \mathrm{m}$, roughness length $z_{0}=0.05 \mathrm{~m}$.

stability; it is closer to the receptor with increasingly convective stratification. Moreover, under convective stratification, the footprint is less skewed in upstream direction. The overall surface area of influence is in convective than in stable conditions since the boundary-layer stability also influences the lateral footprint dimension (Figure 4).

As a measure for the surface area of influence, the 50\%-level source area was calculated following Schmid (1994), i.e., the smallest possible area that includes $50 \%$ of the total flux or concentration footprint (Figure 5). Again, this figure clearly depicts the dependence of the area of influence on the stability regime: It is largest under stable stratification and decreases under convective conditions. The footprint for concentration measurements extends much further upwind than the flux footprint, especially in neutral and stable stratification. This is consistent with earlier findings by Schmid (1994) and by Rannik et al. (2000).

\section{Model Evaluation}

Due to the unavailability of appropriate experimental data sets, validation strategies for footprint models are still an open question. Therefore, we evaluate the present Lagrangian footprint model indirectly by first assuring that the dispersion model, on which the backward trajectory model LPDM-B is based, is able to reproduce the dispersion process adequately. Secondly, in order to evaluate the footprint estimation from the Lagrangian trajectories (Equations (16) and (18)), a comparison to results of other footprint models is presented (Section 4.2).

\subsection{THE DISPERSION MODEL}

The present Lagrangian footprint model is based on the dispersion model (LPDM) of Rotach et al. (1996). Its central assumption, i.e., the simulation of the velocity PDF (Equation (10)), has been shown to compare favourably with observations under various stability regimes (Rotach et al., 1996). 

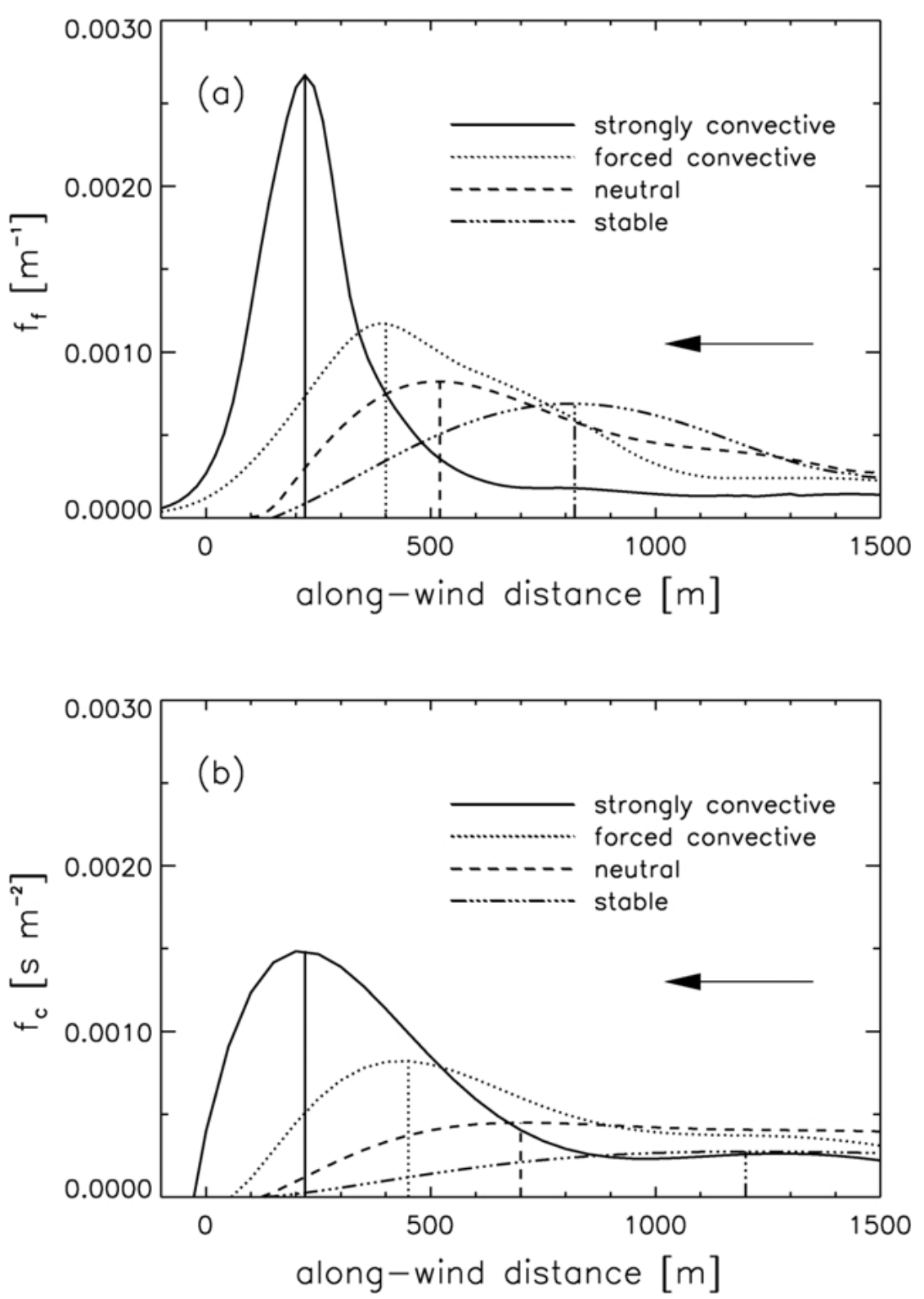

Figure 3. (a) Crosswind-integrated footprint for flux and (b) concentration measurements for four different cases of stabilities as described in Table II. The locations of the respective peaks are indicated by vertical lines. 

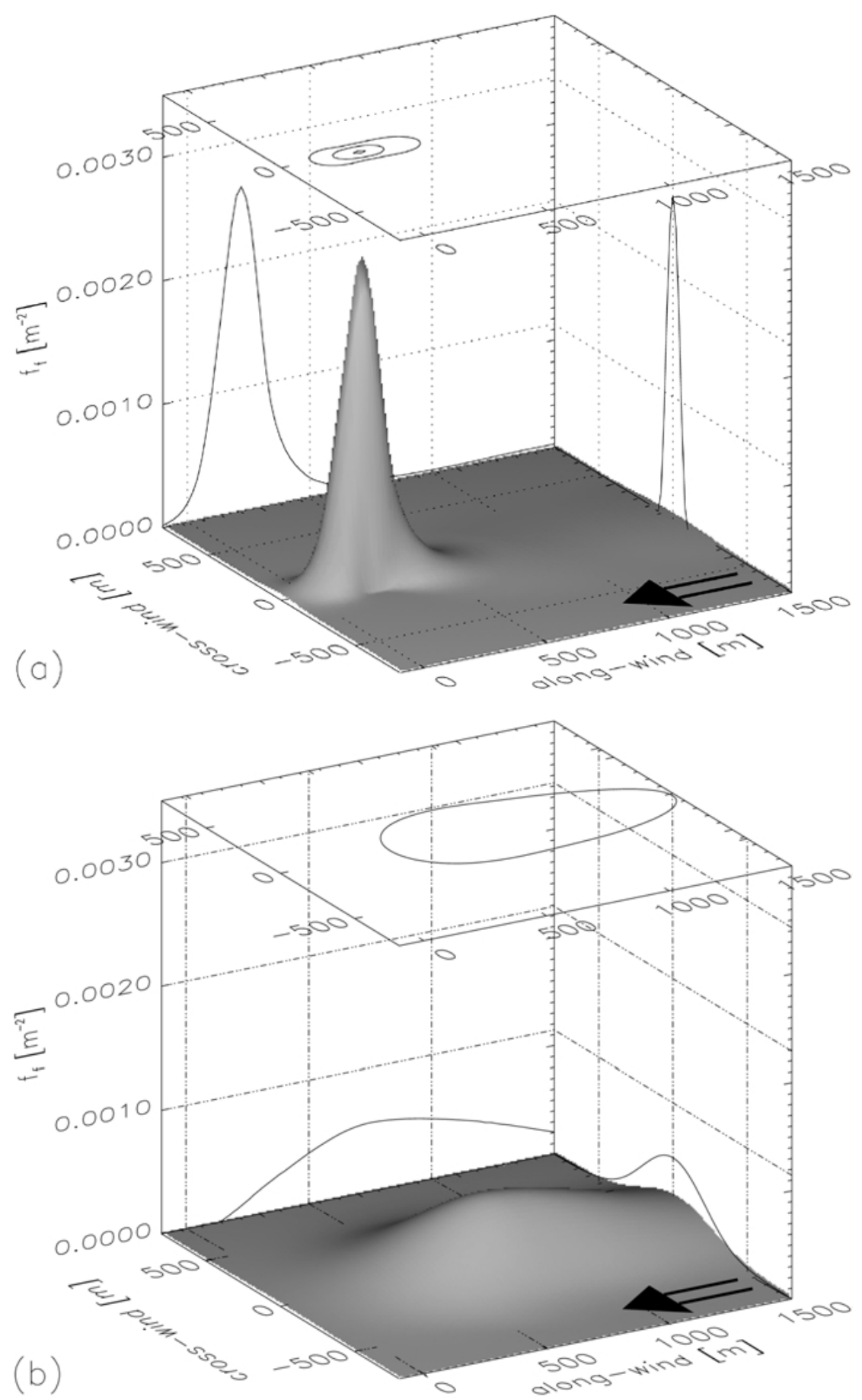

Figure 4. (a) Three-dimensional flux footprint for the strongly convective and (b) the stable case in Table II. 

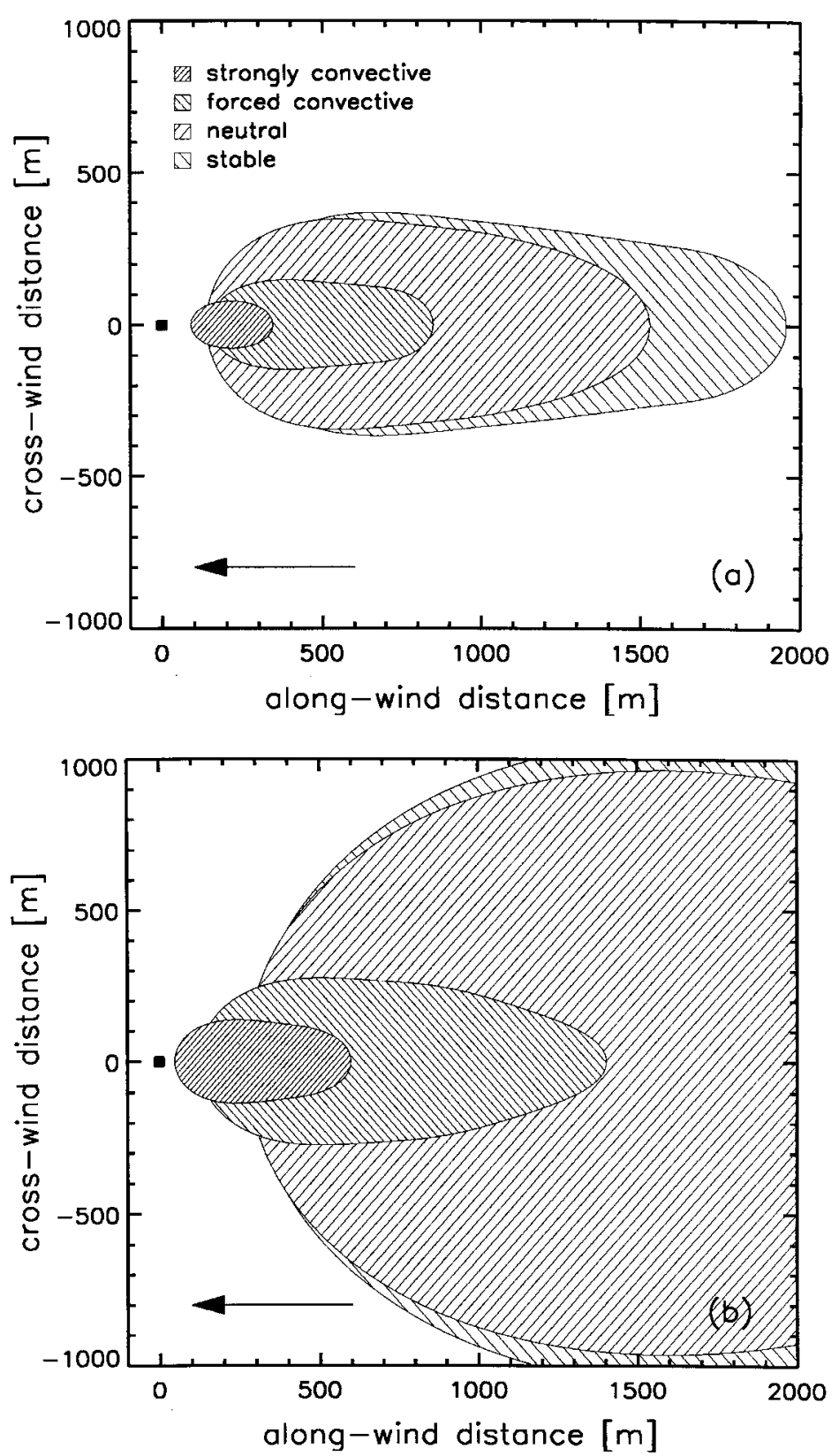

Figure 5. (a) 50\%-level source area for flux and (b) concentration measurements for four different cases of stability as described in Table II. The square indicates the receptor location. 
In free convection conditions, the model is able to reproduce all the features of the dispersion process qualitatively and quantitatively for sources at different heights within the convective boundary layer (see the comparison to the water-tank experiments of Willis and Deardorff (1976, 1978, 1981) in Rotach et al. (1996)). For stability conditions between neutral and strongly convective, the LPDM has been shown to successfully reproduce both field data from a full-scale tracer experiment in Copenhagen and results for LES by Mason (1992). See Rotach et al. (1996) for details. Moreover, PPM, another dispersion model (de Haan and Rotach, 1998), has the LPDM at its core and reproduces the tracer experiments of Copenhagen and Kincaid very well. As far as stable conditions are concerned, no true validation of the LPDM has been published so far. When trying to reproduce the observations from the tracer experiment in Lillestrøm under highly stable conditions, both the LPDM and the PPM are similar to or better than other dispersion models (de Haan and Rotach, 1998), but still hardly satisfactory in their performance. However, this partial 'failure' can be attributed to the turbulence description (similarity approach) rather than to the model formulation itself (Gryning, 1999). Only recently, the LPDM has been used to successfully simulate the stable runs of the Prairie grass experiment, for which Gryning (1999) states that it corresponds to moderately stable conditions and thus similarity-based dispersion models are adequate.

Overall, the dispersion model is validated for a wide range of boundary-layer stabilities and source heights. We can therefore be confident that the dispersion process in the backward model LPDM-B is also adequately described. Moreover, Kljun et al. (1999) have shown that, under convective stratification, the footprints predicted by LPDM-B represent flux observations much better than the simpler Lagrangian model of Thomson (1987), which neglects the skewness in the vertical velocity PDF.

\subsection{COMPARISON OF LPDM-B WITH OTHER FOOTPRINT MODELS}

The estimated footprint functions from the present model are compared with those obtained by an analytical footprint model. The analytical model used in this study is that contained in SAM/FSAM (concentration/flux footprint) after Schmid (1994, 1997) based on an analytical solution of the Eulerian advection-diffusion equation for vertical diffusion using standard surface-layer scaling parameters (Van Ulden, 1978). It is based on K-theory and has a short-range plume model for surfacelayer scaling conditions and ground-level sources (Gryning et al., 1987) at its core, to describe the diffusion characteristics. The latest version of the model uses an approximate equation for the flux footprint function proposed by Horst and Weil (1994). This latter model has been tested against experimental data of trace gas fluxes by Finn (1996), showing that the model predictions usually fell within the uncertainties of the measurements, which, however, were found to be particularly large around neutral stability. For a complete description of SAM/FSAM, the reader is referred to Schmid $(1994,1997)$. 

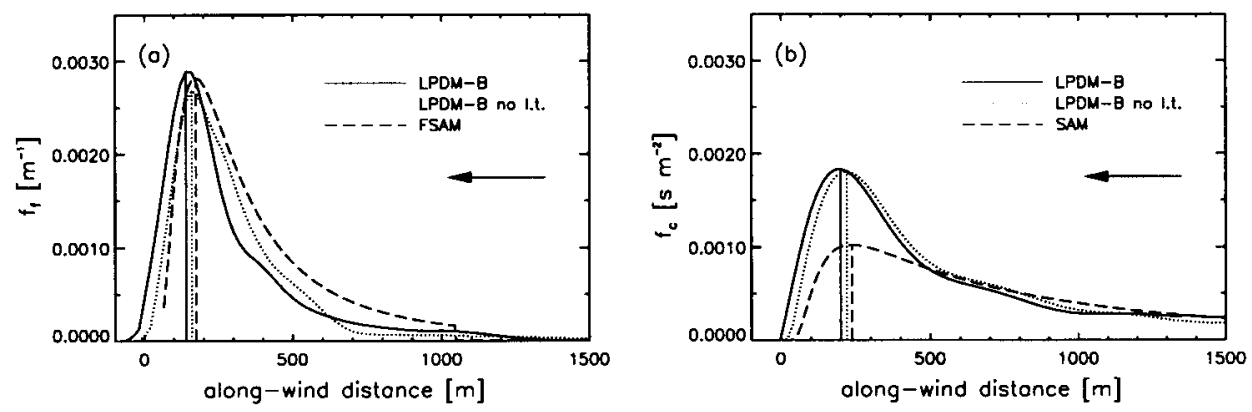

Figure 6. (a) Crosswind-integrated footprint for flux and (b) concentration in forced convective stratification (see Table II) as predicted by LPDM-B with (solid) and without longitudinal turbulence (dotted) and FSAM (dashed) for a receptor height of $20 \mathrm{~m}$.
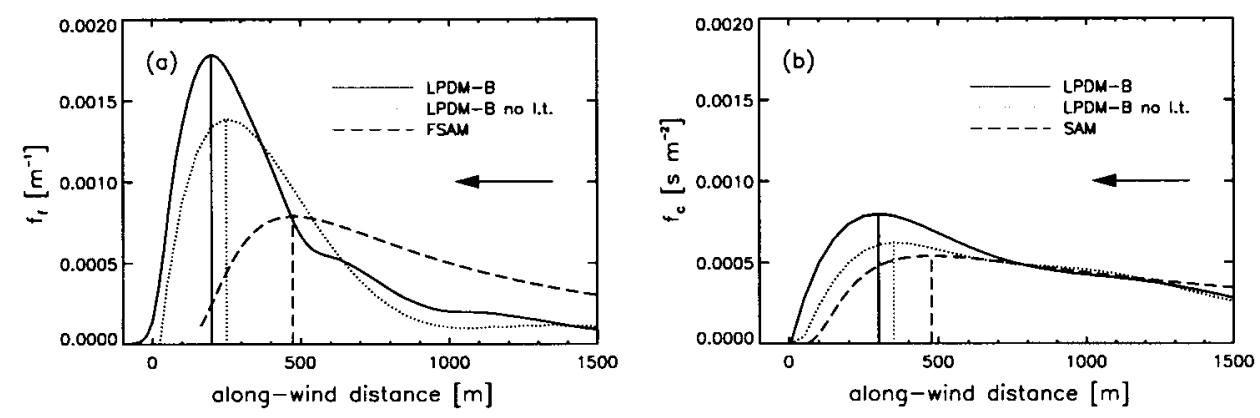

Figure 7. Same as Figure 6 but for neutral stratification.

Like most analytical footprint model, SAM/FSAM are theoretically restricted to moderate stratification within the surface layer. Therefore, the comparison of the two models concentrates on these limits and only the forced convective and the neutral case of Table II for a receptor height of $20 \mathrm{~m}$ and $100 \mathrm{~m}$ were chosen for comparison.

The crosswind-integrated flux footprints for a receptor height of $20 \mathrm{~m}$ under forced convective and neutral stability are presented in Figures $6 \mathrm{a}$ and $7 \mathrm{a}$. For the forced convective case we can see that the flux footprint estimates of the two models show good correspondence in shape and peak location (Figure 6a). However, under neutral stratification, the footprint of LPDM-B reached its peak earlier than FSAM (Figure 7a). Also, the footprint of LPDM-B indicates a small contribution to the measurement from downwind sources.

The underestimation of the contribution of upwind sources close to the receptor and, as a consequence, the shift of the footprint peak by FSAM is partially due to the fact, that analytical footprint models neglect the longitudinal turbulent diffusion. A simulation of LPDM-B with suppressed longitudinal turbulent diffusion was therefore run for the four stability regimes of Table II. Under conditions of forced convection (Figure 7a), the longitudinal turbulence accounts for most part of the difference between the LPDM-B and FSAM. On the other hand, for neut- 
ral stratification (Figure 7a), the relative importance of longitudinal diffusion was somewhat larger but cannot explain the whole discrepancy between the two models. Other factors that may be responsible for the observed difference include the choice of some parameters in FSAM, which are selected for optimal performance under conditions of forced convection (i.e., the most 'common' case) and held constant for all stabilities. This is in contrast to the model by Horst and Weil (1994) and Horst (1999), which dynamically adjusts these parameters for stability (e.g., to describe the mean plume velocity). The peak footprint location predicted by the Horst (1994) model closely matches the current results of LPDM-B (not-shown).

Generally, the behaviour of the concentration footprints was similar to that of the flux footprints. The magnitude and the shape of the footprints of the two models were in good correspondence under neutral and convective stratification for the receptor height of $20 \mathrm{~m}$ (Figures $6 \mathrm{~b}$ and $7 \mathrm{~b}$ ). The footprints predicted by LPDM-B increased earlier than those predicted by SAM which again can partly be explained by the neglected longitudinal turbulence in SAM (Figures 6b, 7b). In general, the correspondence between the two models is somewhat better if concentration footprints are concerned rather than flux footprints. This would suggest the method of calculating the flux estimates from the present LPDM-B simulations could have a major contribution to the observed difference in the flux footprint under neutral conditions. We will come back to this issue when comparing the present simulations with another Lagrangian footprint model below.

Figure 8 shows the flux footprints under neutral and free convection conditions for a receptor height of $100 \mathrm{~m}$. For neutral stratification, there is no formal reason that would inhibit the application of FSAM. Still, with $z_{m} / z_{0}=2000$, and a receptor height in the upper part of the surface layer, this simulation means to push FSAM to its limits. These limits are formally broken under convective conditions since $-z_{m} / L>1$ (Schmid, 1994). As expected, the flux footprints differ considerably for these high receptor locations (Figure 8). The distance of the peak footprint location is different by almost a factor of two or more, and even the simulation of LPDM-B without longitudinal turbulence cannot explain this difference.

Over all, the comparison between the present model and SAM/FSAM is satisfactory in conditions where the latter is valid (Kljun et al., 2000b). However, under neutral conditions non-negligible differences in the location of the peak flux footprint are found and, at the same time, an important contribution of longitudinal dispersion (which is not accounted for in the analytical model). The present Lagrangian model has a much wider range of applicability in terms of stability and receptor height and its algorithm is not subject to strong assumptions concerning the turbulence regime as is the case for the analytical models. Therefore, we may conclude that its footprint estimates are more trustworthy than those from the analytical model. It should be noted that, very often, the limitations of analytical footprint models are too restrictive for real situations. Thus, it is advisable to use a model such as LPDM-B that is valid for a wide range of practical applications. However, we cannot fully validate the footprint estimates without suitable experi- 

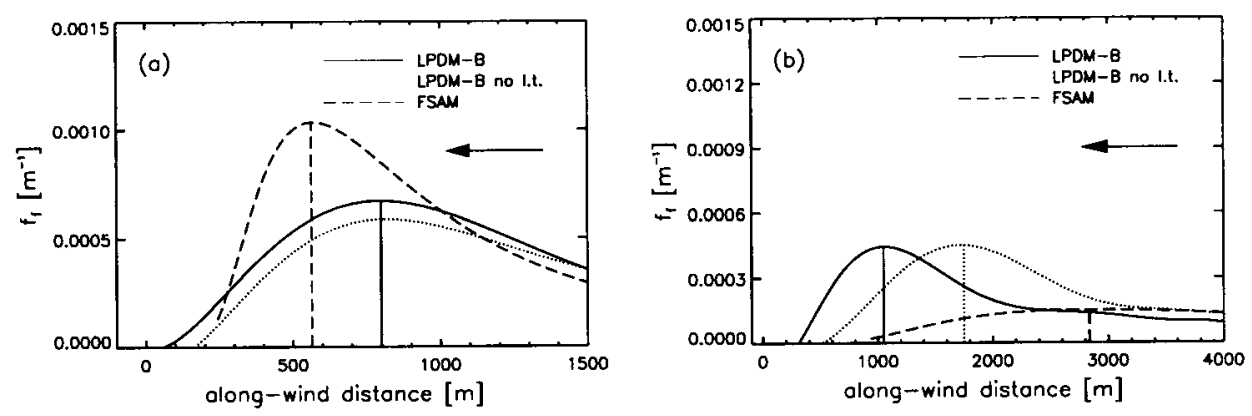

Figure 8. (a) Crosswind-integrated flux footprint for forced convective and (b) neutral stratification (see Table II) as predicted by LPDM-B with (solid) and without longitudinal turbulence (dotted) and FSAM (dashed) for a receptor height of $100 \mathrm{~m}$.
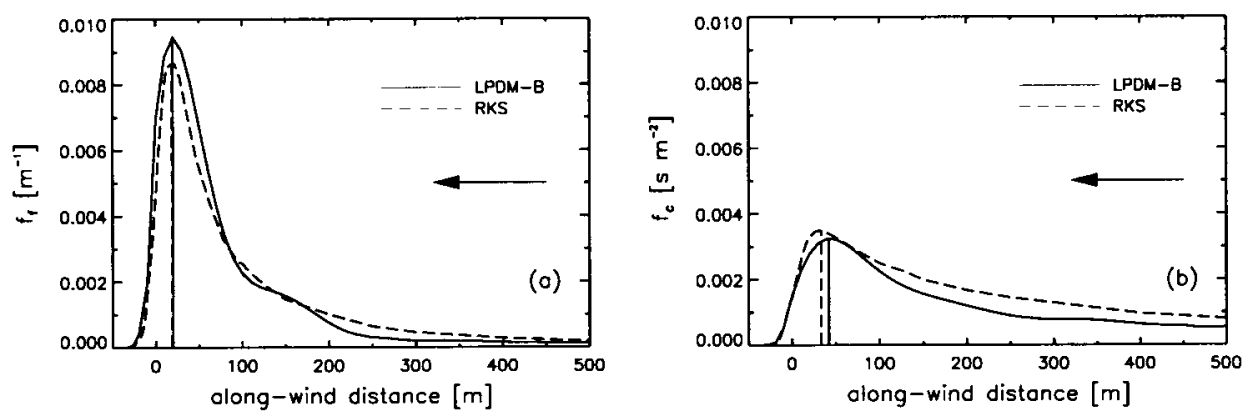

Figure 9. (a) Crosswind-integrated footprint for flux and (b) concentration in neutral stratification as predicted by LPDM-B (solid) and Rannik et al. (2000) (RKS; dashed) for a receptor height of $15 \mathrm{~m}$.

mental data or, at least, another footprint model that allows for high receptor points and stratification outside 'moderate stability'.

As a next step, LPDM-B was compared to the Lagrangian (forward) footprint model of Rannik et al. (2000). This model differs from the present LPDM-B in that it uses a forward approach, and consequently in the method it uses to calculate the fluxes from the particle trajectories. Thus, this comparison provides an independent test of the flux footprint estimation used in our model. Unfortunately, Rannik et al. (2000) present only footprints for neutral stratification. Figure 9 shows flux and concentration footprints for their neutral case (Receptor height $=15 \mathrm{~m}, z_{0}=$ $1.5 \mathrm{~m}$ ). There is excellent agreement between the two models in terms of both the peak location and the shape of the footprint functions. Thus, we conclude that the present flux footprint estimation is robust and the differences as observed in Figure 7a (for another neutral case) are due to differences in the simulation of the diffusion process or some assumptions in the analytical model, rather than due to the flux footprint estimation in the present approach. Yet we stress the point that suitable experimental data are necessary to decide which of the footprint models yields the 'correct' footprint estimates. 


\section{Summary and Conclusions}

A Lagrangian footprint model with a validity range from stable to convective stratification, and for receptor locations ranging over the entire boundary-layer depth is presented. We introduce a statistical treatment of particle touchdowns, the so-called density kernel method. Using this method, a significant reduction of CPU-time was achieved: To obtain the same footprint resolution, the model runs ten times faster than without the kernel method. Thus, the backward Lagrangian model is an efficient tool for footprint predictions. Thanks to a spin-up procedure during initialisation, very good agreement was found between the modelled initial particle velocity distributions and observations. This agreement is important because of the strong influence of the initial velocities on the shape and the peak location of the footprint. The location of the peak footprint contribution is found to differ by up to $30 \%$ under different stability regimes when neglecting the spin-up procedure.

Excellent agreement was found when comparing the present footprint model with a Lagrangian model applying the forward approach. When the footprints predicted by the present Lagrangian model are compared with an analytical model, satisfying correspondence was generally found, as long as the models were applied within the restrictive limits of the analytical model (moderate stability within the surface layer: $-z_{m} / L<1$ and $z_{m}<0.1 z_{i}$ ). However, the flux footprints of the analytical model were clearly further upstream from the receptor location than those predicted by the Lagrangian model. Also, the analytical model was not capable of predicting the small contribution to the footprint downwind of the receptor. These differences are at least partly due to the neglected longitudinal turbulent diffusion of the analytical model.

Analytical models are often used outside their limits of application, as no other simple models for convective conditions exist so far. When the footprints of the Lagrangian model are compared to those of the analytical model applied outside its range (receptor outside the surface layer, or stability too extreme), large differences were found in the peak location and the shape of the footprints. Note, that footprint predictions are often needed for application outside the surface layer (e.g., for use in connection with aircraft measurements or with observations on high towers) and under arbitrary stratification.

Finally, as a backward Lagrangian footprint model, LPDM-B holds the potential to be applied efficiently over inhomogeneous terrain. Even though the present footprint model is constructed from a horizontally homogeneous PDF, it may be used in conjunction with inhomogeneous flow and turbulence fields obtained from numerical models as done in many practical applications of dispersion modelling (e.g., Physick et al., 1994; Luhar and Rao 1994). Furthermore, horizontally inhomogeneous LPDMs are available in the literature for purely Gaussian turbulence (e.g., Thomson, 1987). Even though it may prove to be a major task to extend the present LPDM-B to inhomogeneous conditions, the potential for this is likely one 
of the most important qualities of the present model, considering that footprint estimates are important particularly under inhomogeneous surface conditions.

The present Lagrangian backward footprint model appears to be the first that is able to treat receptor heights throughout the entire boundary layer (as most Lagrangian footprint models do) at the same time as being able to handle a broad range of boundary-layer stratifications. Moreover, with its kernel density estimation, it is much faster than conventional Lagrangian footprint models.

\section{Acknowledgements}

This research was supported by the Swiss Commission for Technology and Innovation (KTI) in the framework of EUROTRAC II (\#3532.1 and \#4092.1), and by a grant from the Terrestrial Carbon Processes program of US-DOE (through a subcontract from Harvard University to HPS). We are grateful to Dr. P. de Haan for helping us with the density kernel estimation.

\section{References}

Baerentsen, J. H. and Berkowicz, R.: 1984, 'Monte Carlo Simulation of Plume Dispersion in the Convective Boundary Layer', Atmos. Environ. 18, 701-712.

de Haan, P.: 1999, 'On the Use of Density Kernels for Concentration Estimations within Particle and Puff Dispersion Models', Atmos. Environ. 33, 2007-2021.

de Haan, P. and Rotach, M. W. : 1998, 'A Novel Approach to Atmospheric Dispersion Modelling: The Puff-Particle Model (PPM)', Quart. J. Roy. Meteorol. Soc. 124, 2771-2792.

Du, S.: 1995, 'Estimation of the Kolmogorov Constant $C_{0}$ ) for the Lagrangian Structure Function, Using a Second-Order Lagrangian Model of Grid Turbulence', Phys. Fluids 7, 3083-3090.

$\mathrm{Du}, \mathrm{S}$.: 1997, 'Universality of the Lagrangian Velocity Structure Function Constant $\left(C_{0}\right)$ across Different Kinds of Turbulence', Boundary-Layer Meteorol. 83, 207-219.

Finn, D., Lamb, B., Leclerc, M. Y., and Horst, T. W.: 1996, 'Experimental Evaluation of Analytical and Lagrangian Surface-Layer Flux Footprint Models', Boundary-Layer Meteorol. 80, 283-308.

Flesch, T. K.: 1996, 'The Footprint for Flux Measurements, from Backward Lagrangian Stochastic Models', Boundary-Layer Meteorol. 78, 399-404.

Flesch, T. K., Wilson, J. E., and Yee, E.: 1995, 'Backward-Time Langrangian Stochastic Dispersion Models and their Application to Estimate Gaseous Emissions', J. Appl. Meteorol. 34, 1320-1332.

Forrer, J. and Rotach, M. W.: 1997, 'On the Turbulence Structure in the Stable Boundary Layer over the Greenland Ice Sheet', Boundary-Layer Meteorol. 85, 111-136.

Gryning, S.-E. 1999, 'Some Aspects of Atmospheric Dispersion in the Stratified Atmospheric Boundary Layer over Homogeneous Terrain', Boundary-Layer Meteorol. 90, 479-494.

Gryning, S.-E., Holtslag, A. A. M., Irwin, J. S., and Sivertsen, B.: 1987, 'Applied Dispersion Modelling Based on Meteorological Scaling Parameters', Atmos. Environ. 21, 79-89.

Horst, T. W.: 1999, 'The Footprint for Estimation of Atmosphere-Surface Exchange Fluxes by Profile Techniques', Boundary-Layer Meteorol. 90, 171-188.

Horst, T. W. and Weil, J. C.: 1992, 'Footprint Estimation for Scalar Flux Measurements in the Atmospheric Surface Layer', Boundary-Layer Meteorol. 90, 171-188.

Horst, T. W. and Weil, J. C.: 1994, 'How Far Is Far Enough?: The Fetch Requirements for Micrometeorological Measurement of Surface Fluxes', J. Atmos. Ocean. Tech. 11, 1018-1025.

Kljun, N., Rotach, M. W., and Schmid, H. P.: 1999, 'Allocation of Surface Sources Using "Backward Trajectory"-Simulations', in preprint, 13th Symposium on Boundary Layers and Turbulence, Dallas, TX, American Meteorological Society, Boston, MA, pp. 187-188. 
Kljun, N., de Haan, P., Rotach, M. W., and Schmid, H. P.: 2000a, 'Footprint Determination in Stable to Convective Stratification Using an Inverse 3D Lagrangian Particle Model', in preprint, 24th Conference on Agricultural and Forest Meteorology, Davis, CA, American Meteorological Society, Boston, MA, pp. 156-157.

Kljun, N., Rotach, M. W., and Schmid, H. P.: 2000b, 'A Lagrangian Footprint Model for Stratifications Ranging from Stable to Convective', in preprint, 14th Symposium on Boundary Layers and Turbulence, Aspen, CO, American Meteorological Society, Boston, MA, pp. 130-132.

Leclerc, M. Y. and Thurtell, G. W.: 1990, 'Footprint Prediction of Scalar Fluxes Using a Markovian Analysis', Boundary-Layer Meteorol. 52, 247-258.

Leclerc, M. Y., Shen, S., and Lamb, B.: 1997, 'Observations and Large-Eddy Simulation Modeling of Footprints in the Lower Convective Boundary Layer', J. Geophys. Res. 102, 9323-9334.

Luhar, A. K. and Rao, K. S.: 1994, 'Source Footprint Analysis for Scalar Fluxes Measured in Flows over an Inhomogeneous Surface', Air Pollut. Model. Appl. X, 315-322.

Mason, P. J.: 1992, 'Large-Eddy Simulation of Dispersion in Convective Boundary Layers with Wind Shear', Atmos. Environ. 26A, 1561-1571.

Physick, W. L., Noonan, J. A., McGregor, J. L., Abbs, D. J., and Manins, P. C.: 1994, LADM: A Lagrangian Atmospheric Dispersion Model, Technical Report 24, CSIRO Division of Atmospheric Research, Australia, 137 pp.

Rannik, U., Aubinet, M., Kurbanmuradov, O., Sabelfeld, K. K., Markkanen, T., and Vesala, T.: 2000, 'Footprint Analysis for Measurements over a Heterogeneous Forest', Boundary-Layer Meteorol. 97, 137-166.

Rotach, M. W., Gryning, S.-E., and Tassone, C.: 1996, 'A Two-Dimensional Lagrangian Stochastic Dispersion Model for Daytime Conditions', Quart. J. Roy. Meteorol. Soc. 122, 367-389.

Schmid, H. P.: 1994, 'Source Areas for Scalars and Scalar Fluxes', Boundary-Layer Meteorol. 67, 293-318.

Schmid, H. P.: 1997, 'Experimental Design for Flux Measurements: Matching Scales of Observations and Fluxes', Agric. For. Meteorol. 87, 179-200.

Schmid, H. P.: 2002, 'Footprint Modeling for Vegetation Atmosphere Exchange Studies: A Review and Perspective', Agric. For. Meteorol. (Special Issue on FLUXNET), in press.

Schmid, H. P. and Oke, T. R.: 1990, 'A Model to Estimate the Source Area Contributing to Turbulent Exchange in the Surface Layer over Patchy Terrain', Quart. J. Roy. Meteorol. Soc. 16, 965-988.

Schuepp, P. H., Leclerc, M. Y., Macpherson, J. I., and Desjardins, R. L.: 1990, 'Footprint Prediction of Scalar Fluxes from Analytical Solutions of the Diffusion Equation', Boundary-Layer Meteorol. 50, 355-373.

Shaw, R. H., Tavangar, J., and David, P. W.: 1983, 'Structure of the Reynolds Stress in a Canopy Layer', J. Clim. Appl. Meteorol. 22, 1922-1931.

Thomson, D. J.: 1987, 'Criteria for the Selection of Stochastic Models of Particle Trajectories in Turbulent Flows', J. Fluid Mech. 180, 529-556.

Van Ulden, A. P.: 1978, 'Simple Estimates for Vertical Diffusion from Sources near the Ground', Atmos. Environ. 12, 2125-2129.

Willis, G. E. and Deardorff, J. W.: 1976, 'A Laboratory Model of Diffusion into the Convective Planetary Boundary Layer', Quart. J. Roy. Meteorol. Soc. 102, 427-445.

Willis, G. E. and Deardorff, J. W.: 1978, 'A Laboratory Study of Dispersion from an Elevated Source within a Modelled Convective Planetary Boundary Layer', Atmos. Environ. 12, 1305-1311.

Willis, G. E. and Deardorff, J. W.: 1981, 'A Laboratory Study of Dispersion from a Source in the Middle of the Convective Layer', Atmos. Environ. 15, 109-117.

Wilson, J. D. and Swaters, G. E.: 1991, 'The Source Area Influencing a Measurement in the Planetary Boundary Layer: The "Footprint" and the "Distribution of Contact Distance", , Boundary-Layer Meteorol. 55, 25-46. 